\title{
LOCAL FEATURE MATCHING USING ENTROPIC GRAPHS
}

\author{
Huzefa Neemuchwala*†, Alfred Hero*†, Paul Carson*\#, Charles Meyer ${ }^{* \#}$ \\ University of Michigan, Ann Arbor \\ Department of Biomedical Engineering*, Department of EECS ${ }^{\dagger}$, Department of Radiology \# \\ Ann Arbor, MI 48109
}

\begin{abstract}
We present a general framework for image discrimination based on identifying small, localized differences between images. Our novel matching scheme is based on an alternate information divergence criterion, the Rényi $\alpha$-entropy. The minimum spanning tree (MST) is used to derive a direct estimate of $\alpha$-entropy over a feature set defined by basis features extracted from images using independent componenet analysis (ICA). The MST provides a stable unbiased estimate of local entropy to identify sites of local mismatch between images. Sub-image blocks are ranked over a set of local deformations spanning small image regions. We demonstrate improved sensitivity to local changes for matching and registration and provide a framework for tracking features of interest in images.
\end{abstract}

\section{INTRODUCTION}

The ability to discriminate differences between images with sensitivity to local differences is pivotal to any image matching algorithm. The principal objective of this paper is to introduce a general framework to identify local deformation in images. Such a framework could be extended to (1) enhance registration performance by making it more sensitive to local mismatch, (2) automatically track features of interest, such as tumors in brain or microcalcifications in breast across temporal image sequences, (3) reliably match or register small images or image regions so as to improve disease diagnosis by locating and identifying small pathological changes in medical image volumes and (4) automate control point placement to initiate registration. Most image registration techniques dwell on similarity measures calculated over some feature set $\left\{X_{i}, Y_{i}\right\}$ derieved from $i=$ $1, \ldots, M \times N$ positions in the reference and target images $I_{\text {ref }}$ and $I_{t a r}$ respectively. Previous work in these techniques has been limited to simple pixel based mutual information (MI) and pixel correlation techniques. In [1], local measures of MI outperform global MI in the context of

Corresponding author: Huzefa Neemuchwala, R3315 Kresge III, 200 Zina Pitcher Pl., Ann Arbor, MI 48109-0553, hneemuch@umich.edu. This work was supported in part by NIH grant 1P01CA87634. adaptive grid refinement for automatic control point placement. However, the sensitivity of local MI deteriorates rapidly as the size of the image window decreases below $40 \times 40$ pixels in 2D. The main constraints on these algorithms, when localizing differences, are (1) limited feature resolution of single pixel intensity based features, and (2) histrogram estimators $h(X, Y)$ of joint probability density $f(X, Y)$ are noisy when computed with a small number of pixel features and are thus poor estimators of $f(X, Y)$ used by the algorithm to derieve joint entropy $H(X, Y)$.

Reliable identification of subtle local differences within images, is key to improving registration sensitivity and accuracy. Stable unbiased estimates of local entropy are required to identify sites of local mismatch between images. These estimates play a vital role in successfully implementing local transformations. Previously [2], we addressed the issue of global registration and demonstrated that entropic graph methods could achieve lower mean square errors in ultrasound image registration. Here, we attempt to improve registration sensitivity by identifying local mismatch between images. Our joint registration model includes a rich ICA derived feature set that provides a continuous representation of images and fast converging direct entropy methods to calculate the joint entropy over these features.

This paper is organized as follows: section 2 introduces the ICA basis feature set, section 3 describes $\alpha$-entropy based on the MST, section 4 discusses the image matching framework, sections 5 and 6 present the results and conclusions.

\section{ICA BASIS FEATURES}

We adopt a basis extracted from an independent components analysis of the image. The ICA basis are adapted so as to best account for the image structure in terms of a collection of statistically independent components. In ICA, an optimal basis is found which decomposes the image or sub-image block $X_{i}$ into a small number of approximately statistically independent components $\left\{S_{j}\right\}$ :

$$
X_{i}=\sum_{j=1}^{d} a_{i j} S_{j} .
$$


The basis elements $\left\{S_{j}\right\}$ are selected from an over-complete linearly dependent basis using randomized selection over the training set of images in a representative database. For image $i$ the feature vectors $Z_{i}$ are defined as the coefficients $\left\{a_{i j}\right\}$ in (1) obtained by projecting the image onto the basis [3]. Given the ICA basis and a pair of to-be-registered $N \times M$ images, coefficient vectors are extracted by nonlinearly projecting each $8 \times 8$ subimage in the images onto the basis set. FastICA [3] was used to implement ICA.

\section{MST ENTROPY ESTIMATOR}

The MST method is a graph-theoretic technique, which determines the dominant skeletal pattern of a point set by mapping the shortest path of nearest neighbor connections. Given a set $Z_{n}=\left\{z_{1}, z_{2}, \ldots ., z_{n}\right\}$ of $n$, i.i.d vectors $Z_{i}$ in $\mathbf{R}^{\mathbf{d}}$ each with density $f$, a spanning tree is a connected acyclic graph which passes through all coordinates associated with $Z_{n}$. In this graph all $n$ points are connected by $n-1$ edges $\left\{e_{i}\right\}$. For a given real weight exponent $\gamma \in(0,1)$ the minimum spanning tree is the spanning tree which minimizes the total edge weight:

$$
L\left(Z_{n}\right)=\min _{e \in T} \sum_{e}\|e\|^{\gamma}
$$

where $\|e\|$ denotes Euclidean (L2) norm of the edge. The overall length of the MST can be used to construct a strongly consistent estimator of entropy $[4,5]$.

It can be shown [5] that the length function when normalized by $\sqrt{n}$ produces sequences that converge within a constant factor to the alpha entropies with $\alpha=1 / 2$. More generally, for i.i.d. points in $\Re^{d}$, by changing the value of $\gamma$ in (2), one can change the convergent limit to the $\alpha$-entropy where $\alpha=(d-\gamma / d)$. The MST is called an entropic spanning graph as its normalized log-length converges (a.s.) within a constant to an $\alpha$-entropy. Specifically, the Rényi entropy estimator

$$
\hat{H}_{\alpha}\left(Z_{n}\right)=1 /(1-\alpha)\left[\ln L\left(Z_{n}\right) / n^{\alpha}-\ln \beta_{L, \gamma}\right],
$$

is an asymptotically unbiased and almost surely consistent estimator of the $\alpha$-entropy of $f$ where $\beta_{L, \gamma}$ is a constant bias independent of $f$ [5].

As contrasted with density based estimates of entropy, the MST estimator completely by-passes the complication of choosing and fine-tuning parameters such as histogram bin size, density kernel width, complexity and adaptation speed. For large dimensions the MST can be implemented when histograms cannot, due to the "curse of dimensionality". For small regions, the sparseness of the histogram causes poor accuracy of the derived entropy estimate.

\section{DEFORMATION LOCALIZATION}

Iterative registration algorithms apply transformations to a sequence of images while minimizing some objective function. We demonstrate the sensitivity of our technique by tracking deformations that correspond to small perturbations of the image. These perturbations are recorded by the change in the mismatch metric.

Global deformations reflect a change in imaging geometry and are modeled as global transformations on the images. However, global similarity metrics are ineffective in capturing local deformations in medical images that occur due to physiological or pathological changes in the specimen. Typical examples are: change in brain tumor size, appearance of microcalcifications in breast, non-linear displacement of soft tissue due to disease and modality induced inhomogeneties such as in MRI and nonlinear breast compression in XRay mammograms. Most registration algorithms will not be reliable when the size of the mismatch site is insufficiently small, typically $(m \times n) \leq 40 \times 40$ [1].

We note here that the $\alpha$-entropy function is a similarity function akin to the standard MI function. It scores image regions with respect to some reference image region over a set of transformations. Of interest is the magnitude and location of change in the objective function (here, the joint entropy). With a combination of ICA and $\alpha$-entropy we match sites having as few as $8 \times 8$ pixels.

In fig. 1, multimodal synthesized scan of $\mathrm{T} 1$ and $\mathrm{T} 2$ weighted brain MRI each of size $256 \times 256$ pixels [6] are seen. The original target images shall be deformed locally (see below) to generate a deformed target image.

\subsection{Locally deforming original image using B-Splines}

B-spline deformations are cubic mapping functions that have local control and injective properties [7]. The 2D uniform tensor B-spline function $F$, is defined with a $4 \times 4$ control lattice $\phi$ in $\mathbf{R}^{2}$ as:

$$
F(u, v)=\sum_{i=0}^{3} \sum_{j=0}^{3} B_{i}(u) B_{j}(v) \phi_{i j},
$$

where $0 \leq u, v \leq 1, \phi_{i j}$ is the spatial coordinates of the lattice and $B_{i}$ are the standard B-Spline basis functions.

Given that the original images have $256 \times 256$ pixels, we impose a $\operatorname{grid}(\Phi)$ of $10 \times 10$ control points on $I_{t a r}$. Since the aim is to deform $I_{t a r}$ locally, not globally, we select a subgrid $(\phi)$ of $4 \times 4$ control points in the center of $I_{\text {tar }}$. We then diagonally displace, by $\ell=10 \mathrm{~mm}$, only one of the control points in $\phi$, to generate deformed grid $\phi_{d e f} . I_{t a r}$ is then reconstructed according to $\phi_{d e f}$. The induced deformation is measured as $\left\|\phi_{d e f}-\phi\right\|$. Fig.1.b and 1.c show the resultant warped image and difference image, $I_{\text {tar }}-T\left(I_{t a r}\right)$. For smaller deformations, $\Phi$ is a finer grid 
of $20 \times 20$ points, from which $\phi$ is picked. A control point in $\phi$ is then displaced diagonally by $\ell=1,2, \ldots 10$ to generate $\phi_{\text {def }}$. When $\ell \leq 3$, noticeable deformation spans only $8 \times 8$ pixels.

\subsection{Feature discrimination algorithm}

We generate a $d$-dimensional feature set $\left\{Z_{i}\right\}_{i=1}^{m \times n}, m \times$ $n \geq d$ by sequentially projecting sub-image block (window) $\left\{\Gamma_{j}\right\}_{j=1}^{M \times N}$ of size $m \times n$ onto a $d$-dimensional basis function set $\left\{S_{k}\right\}$ extracted from the MRI image. Raster scanning through $I_{\text {ref }}$ we select sub-image blocks $\left\{\Gamma_{i}^{r e f}\right\}_{i=1}^{M \times N}$. For this simulation exercise, we pick only the sub-image block $\Gamma^{\text {tar }}$ from $T\left(I_{t a r}\right)$ corresponding to the particular pixel location $k=(128,128) . \Gamma_{128,128}^{t a r}$ corresponds to the area in $I_{\text {tar }}$ where the B-Spline deformation has been applied.

The size of the ICA basis features is $8 \times 8$, i.e. $d=64$. The MST is constructed over the joint feature set $\left\{Z_{i}^{r e f}, Z_{j}^{\text {tar }}\right\}$. When suitably normalized with $1 / n^{\alpha}, \alpha=0.5$, the length of the MST is an estimate of $H_{\alpha}\left(Z_{i}^{r e f}, Z_{j}^{\text {tar }}\right)$. We score all the sub-image blocks $\left\{\Gamma_{i}^{r e f}\right\}_{i=1}^{M \times N}$ with respect to the subimage block $\Gamma_{128,128}^{\operatorname{tar}}$. Let $O_{\ell}$ be the resultant $M \times N$ matrix of scores, at deformation $\ell$. The objective function surface $O_{\ell}$ is a similarity map beween $\left\{\Gamma^{r e f}\right\}_{i=1}^{M \times N}$ and $\Gamma^{t a r}$. When two sites are compared, the resulting joint probability distribution depends on the degree of mismatch. The best match is detected by searching for the region in the $I_{\text {ref }}$ that corresponds to $\Gamma^{t a r}$ as determined by the MST length. As opposed to the one-to-all block matching approach adopted here, one could also perform a block-by-block matching, where each block $\Gamma_{i}^{r e f}$ is compared with its corresponding block $\Gamma_{i}^{t a r}$. The particular approach adopted should depend on the application at hand. Also, for window size $8 \times 8$, the projection of each window on the $8 \times 8$ basis gives one $64 \mathrm{D}$ coefficient per window. Then the MST length is simply the Euclidean distance between the two 64D coefficients.

\section{RESULTS}

Fig.1 (d,e,f) shows $O_{10}$ for $m \times n=8 \times 8,16 \times 16$ and $32 \times 32$. Similar maps can be generated for $\ell=\ell_{1}, \ell_{2}, \ldots \ell_{p}$. The gradient $\nabla(O)=O_{\ell_{1}}-O_{\ell_{2}}$ reflects the change in $H_{\alpha}$, the objective function, when $I_{\text {tar }}$ experiences an incremental change in deformation, from $\ell=\ell_{1} \rightarrow \ell_{2}$. This gradient, at various sub-image block size is seen in Fig.1 (g,h,i), where $\ell_{1}=0$ and $\ell_{2}=10$. For demonstration purposes, the size of the deformation applied to $I_{t a r}$ is large. Smaller deformations generated using a control grid spanning only $40 \times 40$ pixels are used to generate Fig. $1(\mathrm{~m})$. It shows the ratio of the gradient of the objective function:

$$
R=\frac{\frac{1}{(m \times n)} \sum_{i=1}^{m \times n}|\nabla(O(i))|}{\frac{1}{(M \times N-m \times n)} \sum_{i=1}^{M \times N-m \times n}|\nabla(O(i))|},
$$

over the deformation site $\mathrm{v} / \mathrm{s}$ background in the presence of additive gaussian noise.

Fig. $1(\mathrm{j}, \mathrm{k}, 1)$ shows the similarity map $O_{\ell}$ when constructed using a histogram estimate of joint entropy calculated over subimage size $m \times n$, as:

$$
M I=\sum_{x=0}^{255} \sum_{y=0}^{255} f(x, y) \log \left(\frac{f(x, y)}{f(x) f(y)}\right) .
$$

At lower sub-image sizes, the estimate has bias and several local minima even under noise free conditions. It is thus unsuitable for detection of local deformation of $I_{t a r}$.

\section{CONCLUSION}

We have presented a feature discrimination technique that registers the local changes in the images, even under low SNR. Our technique has a combination of high resolution features for image discrimination and a fast converging stable entropy estimation technique based on the MST. Together they provide ability to score images over a set of local transformations. We applied these techniques to identify sites of local non-linear deformation.

\section{REFERENCES}

[1] H. Park and C. Meyer, "Grid refinement in adaptive non-rigid registration," Lecture Notes in Computer Science, MICCAI 2003 (to appear), 2003.

[2] H. Neemuchwala, A. O. Hero, and P. Carson, "Image matching using alpha-entropy measures and entropic graphs," European Journal of Signal Processing, Accepted, June 2003.

[3] A. Hyvärinen and E. Oja, "Independent component analysis: algorithms and applications," Neural Networks, vol. 13, no. 4-5, pp. 411-430, 1999.

[4] A.O. Hero, J. Costa, and B. Ma, "Convergence rates of minimal graphs with random vertices," IEEE Trans. on Inform. Theory, vol. submitted, 2002.

[5] A.O. Hero, B. Ma, O. Michel, and J. Gorman, “Applications of entropic spanning graphs," IEEE Signal Processing Magazine, vol. 19, no. 5, pp. 85-95, Sept. 2002.

[6] C. A. Cocosco, V. Kollokian, R. K. S. Kwan, and A. C. Evans, "Brainweb: Online interface to a 3D MRI simulated brain database," NeuroImage, vol. 5, no. 4, 1997.

[7] Y. Choi and S. Lee, "Injectivity conditions of 2D and 3D uniform cubic B-Spline functions," Graphical Models, vol. 62, pp. 411-427, 2000. 


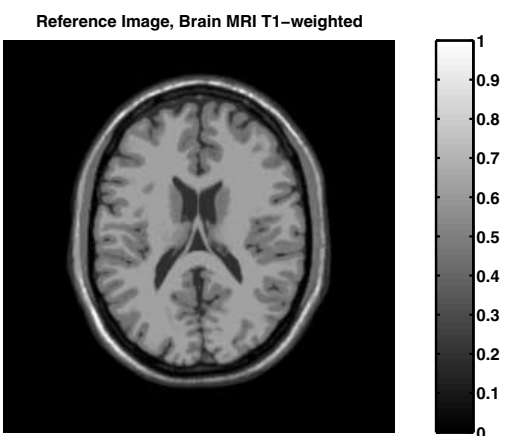

(a) $I_{r e f}$
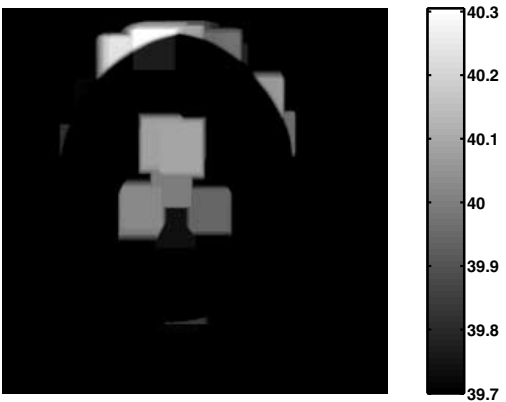

(d) $O_{10}=H_{\alpha}(X, Y): 32 \times 32$ window
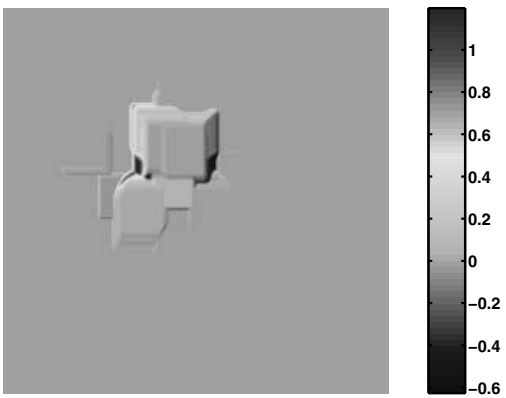

(g) Local $\nabla\left(H_{\alpha}\right)=O_{10}-O_{0}: 32 \times 32$ window

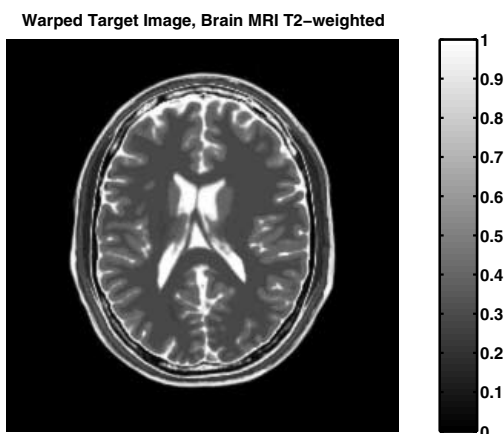

(b) $T\left(I_{\text {tar }}\right)$

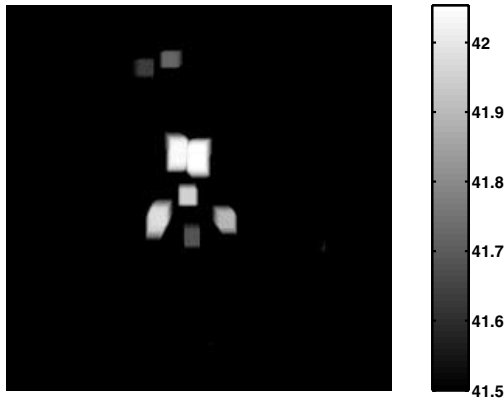

(e) $O_{10}=H_{\alpha}(X, Y): 16 \times 16$ window

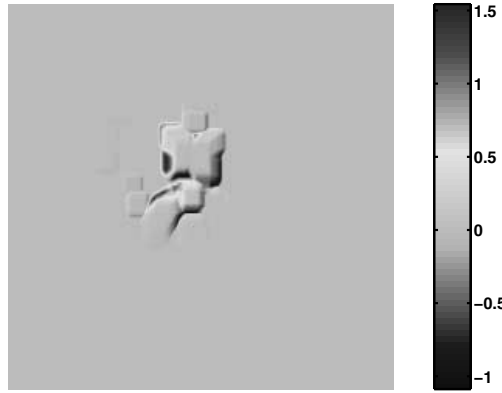

(h) Local $\nabla\left(H_{\alpha}\right)=O_{10}-O_{0}: 16 \times 16$ window

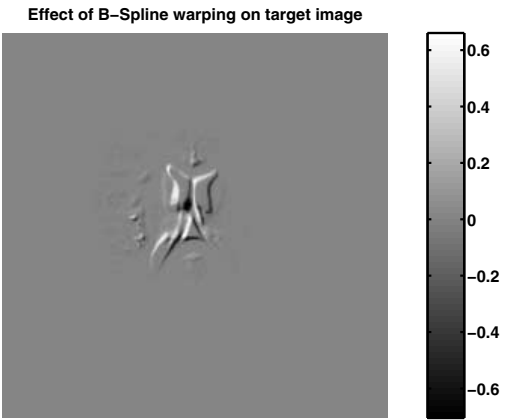

(c) Difference Image $\left(I_{t a r}-T\left(I_{t a r}\right)\right)$

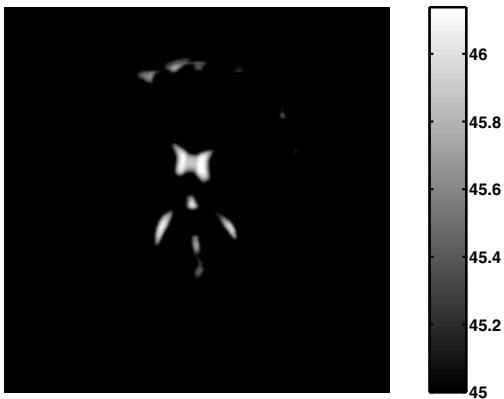

(f) $O_{10}=H_{\alpha}(X, Y): 8 \times 8$ window

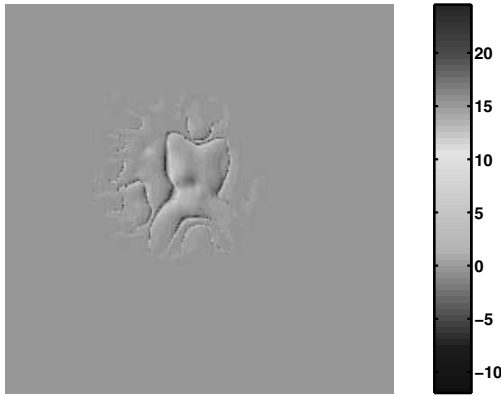

(i) Local $\nabla\left(H_{\alpha}\right)=O_{10}-O_{0}: 8 \times 8$ window

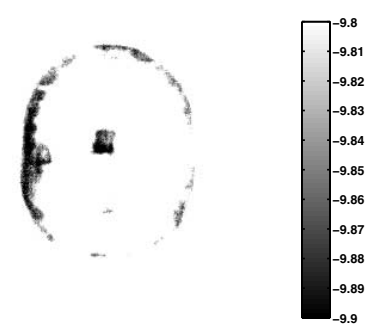

(j) Shannon MI $O_{10}=$ $M I(X, Y): 32 \times 32$
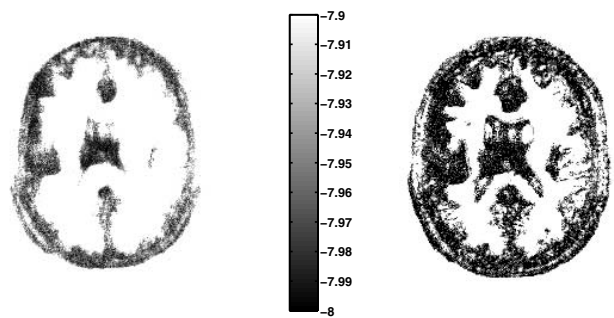

(k) Shannon MI $O_{10}=$ $M I(X, Y): 16 \times 16$
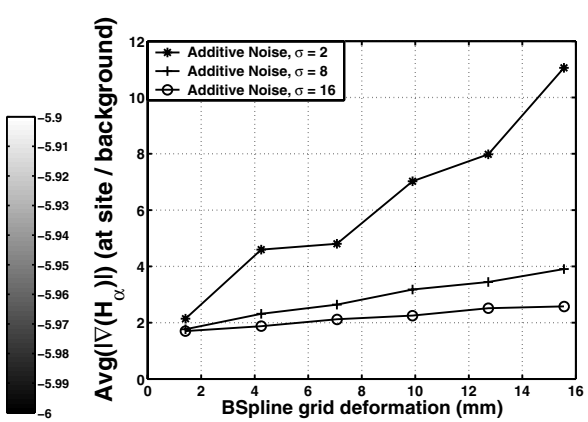

(m) SNR of $\left|\nabla\left(H_{\alpha}\right)\right|$

Fig. 1. B-Spline deformation on MRI images of the brain. (a) Reference image, (b) Warped target (c) True Deformation, (d) $O_{10}=H_{\alpha}$ as seen with a $32 \times 32$ window, (e) $16 \times 16$ window and (f) $8 \times 8$ window. (g) $\nabla(O)=\nabla\left(H_{\alpha}\right)=O_{10}-O_{0}$ as seen with a $32 \times 32$, (h) $16 \times 16$ and (i) $8 \times 8$ window. (j,k,l) Shannon MI using pixel intensity and histograms. (m) Ratio of $\nabla\left(H_{\alpha}\right)=\nabla O$ calculated over deformation site v/s background image for smaller deformation spanning $m \times n \geq 8 \times 8$. 